\title{
Improvement User Interface in Virtual Learning Environment
}

\author{
Siamak Barzegar, Mohammad Shojafar, \\ M.R.Keyvanpour Islamic Azad University of Qazvin, Iran
}

\begin{abstract}
Designing user interface has special importance in Virtual Learning. Considerable portion of students' time is spent in attempting to learn system, work with present lists in user interface and so forth. Perhaps virtual education system has several computational powers, but it lacks adequate user interface. Then, using system would be difficult and user may encounter many confuses during the work and would be unsuccessful in reaching their goals. These are results of unfamiliarity with fundamental principles and in user interface design and disusing them in generating virtual learning systems. Thus, in any virtual learning system design, it is necessary to consider fundamental principles and rules of the user interface design in such system, and in this manner, in this research an adequate user interface is suggested for e-learning Environment in which has been evaluated with regard to these fundamental principles and rules. Evaluations have been done on this interface show its desirable ability to attract users' satisfaction.
\end{abstract}

\section{Introduction}

In science and technology world and with quick and interesting advance in e-learning field, user interface is the most important factor in quality control, attracting users' and service giving's confidence. Interface is a very important component of any industry and technology. Information technology also is no exception.

In the past, computer software was designed with little regard for the user, so the user had to somehow adapt to the system. This approach to system design is not at all appropriate today-the system must adapt to the user.

This is why design principles are so important. Computer users should have successful experiences that allow them to build confidence in themselves and establish self-assurance about how they work with computers. Their interactions with computer software should be "success begets success." Each positive experience with a software program allows users to explore outside their area of familiarity and encourages them to expand their knowledge of the interface. Well-designed software interfaces, like good educators and instructional materials, should build a "Teacher-Student" relationship that guides users to learn and enjoy what they are doing. Good interfaces can even challenge users to explore beyond their normal boundaries and stretch their understanding of the user interface and the computer. When you see this happen, it is a beautiful experience. UI Designer Should understand and aware of the user's mental model and the physical, physiological, and psychological abilities of users.

This is why design principles are so important. Computer users should have successful experiences that allow them to build confidence in themselves and establish self-assurance about how they work with computers. Their interactions with computer software should be "success begets success." Each positive experience with a software program allows users to explore outside their area of familiarity and encourages them to expand their knowledge of the interface. Well-designed software interfaces, like good educators and instructional materials, should build a "Teacher-Student" relationship that guides users to learn and enjoy what they are doing. Good interfaces can even challenge users to explore beyond their normal boundaries and stretch their understanding of the user interface and the computer. When you see this happen, it is a beautiful experience. UI Designer Should understand and aware of the user's mental model and the physical, physiological, and psychological abilities of users.

Interface design principles represent high-level concepts and beliefs that should be used to guide software design. You should determine which principles are most important and most applicable for your systems and then use these principles to establish guidelines and determine design decisions.

Designing the user interfaces for Virtual learning Environment must be in a way that users do not sense the lack of physical Attending of the Teacher. Designing an appropriate use interface will make it possible for virtual learning to be just the same as learning with other users in a class and with the presence of a Teacher and the expected efficiency will be just as same as what is expected in a real educational class. In order to make it possible for the users to achieve a more complete understanding of the designed system, For VLE Design, Designer should consider the physical conditions of the environment and spiritual conditions and the user's capabilities.

Many applications and addressed subjects in this technology are based on interface in order to develop, implement and even being accept among their addressees. Working with complicated and confused user interface has led some to avoid using computer 
systems. As a result, it should consider concerns and demands of users in software and systems to respond users' request.

According to increasingly virtual education environment growth and their importance, it is required to present an adequate and acceptable user interface for users in virtual education Environment, based on principles, rules and regulations of user interface design.

Users interface design for virtual educations learning must be completely communicative with virtual learning environment. A good user interface design provides conditions for users in such way that users' education become considered virtually as the same as a real class and they may feel they interact in a real educational environment. For more over, users' efficiency in a virtual Environment must become completely the same as their efficiency in a real educational class. To more understand and familiarity of users with a designed system, in addition to mention physical Environmental conditions, it should also consider their mental conditions and abilities.

In second part, general study of virtual education and its presentation on sites have been discussed. In third part, Series of fundamental rules and golden principles for a user interface in virtual Environments and virtual education is introduced. In fourth part, with respect to fundamental rules and principles, an adequate user interface for virtual education Environment is suggested, and in fifth part, suggested user interface is being evaluated and in the sixth part, conclusions of the research has been covered.

\section{Virtual Education}

As a definition it may say virtual university and virtual education are being applied to any course and training in which are implemented in a way rather than face to face traditional methods. Course contents may transfer via Internet and or using interactive video and animated images. Also cable or satellite TVs may become transferable media for these courses or it is possible to present syllabus on CD or DVD and or video tape or used combination of the above cases.

Virtual university is a complex university in which students may enroll through Internet, select considered courses among presented courses, participate course sessions, take an exam and communicate with supervisor and class mates. Virtual university also provides tools to system and professors in order to do their tasks via Internet. The university provides managerial tools for manager such as course presenting, selecting supervisor, term time table, financial management. The professor also has tools that may present the course, take an exam and enter marks for students [1].
Today, with modern information and communicative technologies development, business method and daily activities, relating to others, access to information and generally all the aspects of human life become largely evolutionally changed and gaining knowledge are not exceptional among.

Although mankind has used technology and tools for educational and learning services, but maybe education and learning have been never adapted to considerable changes due to using modern information and communicative technologies such as Internet, Compact Disk and multimedia systems. These technologies, mainly with purpose of improving quality level for presenting educational services and also goals such as extending number of addressees, enable professors and educational institutions (and even companies and business centers) to transfer their considered subjects and contents to students, customers and generally addressees, cost-effectively and in shortest time [2].

Virtual learning or virtual education is the same. Now, we should define an environment that it is useful for virtual educating.

Here, we present five main specific of Virtual Learning environment from Dillenburg [3] that they are:

A virtual learning environment is a designed information space.

A virtual learning environment is a social space.

Virtual leaning environments are not restricted to distance education.

A virtual learning environment integrates multiple tools.

The virtual environment overlaps with the physical environment.

We explain more about these specifications of VLE in sub section of this section.

\subsection{A virtual learning environment is a designed information space}

Any Web site is a built information space. In many cases though, this information space is just spaghetti of HTML files. We refer to the 'architecture' of information instead of 'Structure' or 'organization' of information in order to emphasize the fact that the structure Results from analyzing the functional requirements of the environment. For learning Environments, the functional requirements are numerous and have not been yet systematically studied. Here are a few examples:

Using information in educational interactions. For answering simple questions such as "Give me an example of..." or "Give me an argument against..." information must be stored in tables (databases producing dynamically Web pages) or in HTML files enriched with meta-information.

Multi-authoring. The information stored in a virtual learning environment is produced by many 
authors: several teachers, students, domain experts, Who is authoring what must be explicitly stored in the system for developing mechanisms for sharing objects (e.g. «locking» an object when somebody is editing it) and workflow techniques (e.g. the document produced by $\mathrm{X}$ must be sent for approval to $\mathrm{Y}$ and $\mathrm{Z}$ before to be displayed).

Indicating information source. Web information without explicit information regarding to (the authority of) its author will soon have no more value

Maintaining information. When Web sites grow, if information has not been carefully structured, maintenance becomes very heavy: maintaining links, removing obsolete information, The cost of maintaining a Web site may become higher that the costs of creating the site! And despite this, it is rarely included in the budget.

Following technical evolution. The effort devoted to developing Web sites has to survive the current technology. Structuring information and adding Meta information increases the potential of reusability of information.

Sharing information with the world. Education would benefit from richer possibilities to share information outside the environment. There are currently efforts to establish worldwide accepted 'resource description formats' and to specialize them for educational purposes.

Today's use of 'virtual learning environment' is not restricted to well-structured information spaces, but I expect that this criterion will become more salient, as content management becomes a main issue for all teachers involved in virtual learning environments. Researchers have to develop a better understanding of the functional relationship between how information is structured and represented and how it can be used in learning activities and interactions.

These points illustrate the fact that developing a school Intranet is a bigger challenge than building on the Internet (Comment by U. Hoppe), but that, at the same time, the Intranet information should be made available for wider communities.

\subsection{A virtual learning environment is a social space}

A book can hardly be described as a learning environment. But, reading a book in a seminar, discussing with other students, writing a summary for the tutor, do constitute a learning environment. Similarly, a set of Web pages does not constitute a virtual learning environment unless there is social interaction about or around the information. This includes synchronous (e.g. chat, MUDs...) versus asynchronous (e.g. electronic mail, forums,) communication, one-to-one versus one-to-many or many-to-many, text-based versus audio and video.
This includes also indirect communication such as sharing objects.

What is specific to virtual environments compared to any information space is that it is populated [4]. The users are inside the information space and see a representation of themselves and/or others in the space. As soon as students see who else is interested by which information, the space becomes inherently social. Researchers have introduced the notion of «place [5] « to emphasize that space as a social impact. Places are "settings in which people interact [6]. "While spaces take their sense from configuration of brick, mortar, wood and glass, places take their sense from configurations of social actions. Places provide what we call appropriate behavioral framing" [7].

The notion of social space opens interesting possibilities that are only explored for a few years. Here are a few examples:

If a student looks for a book, he/she may go to the library and use standard search techniques. A library is a well-structured information space. Besides the library, the set of university offices, in which each professor has its own books, does also constitute an information space, but socially structured. This type of information architecture may be more useful in some cases, for instance if the student does not know much what he/she is looking for.

Social space can be represented explicitly. For instance, students may leave trace of their presence in a room or on a page. Viewing which area has been visited by other students is an indirect mode of interaction.

The social space can be represented per se, for instance by drawing a graph in which students are the nodes and the thickness of the link between two nodes represents the number of e-mail messages between two students [8].

\subsection{Virtual leaning environments are not restricted to distance education}

Web-based education is often associated to distance education, while - in the practice- it is also widely used to support presentable learning. Actually, the difference between distance education and presentable education is fading for several reasons.

Many distances education students do not live far away from the physical school but have tight time constraints (often they work). Asynchronous communication provides them with time flexibility, a growing concern in our society.

Many Web-based courses combine distance and presence, which makes learning environments more robust. Whatever technology is used, all tools have intrinsic limitations. These limitations do, over time, become real obstacles to learning. Even a small amount of co-presence may solve some of the 
problems that can hardly be solved at distance. Examples are activities that require presence such as: launching a new project, complex technical assistance, repairing deep conceptual misunderstanding, negotiation.

These points are important for vocational training, university courses and lifelong learning. In primary and secondary schools, the opposite balance is found: so far, Internet-based activities are there to enrich presentable learning activities, not to replace them. The enrichment can be just an add-on, for instance the teachers' points to Web pages that the students should read. This is not the case for virtual learning environments.

\subsection{A virtual learning environment integrates multiple tools}

A physical learning environment generally integrates courses, resources (libraries), formal communication (boards) and informal communication (cafeteria ...), an administration ...

Similarly, a virtual learning environment integrates a variety of tools supporting multiple functions: information, communication, collaboration, learning and management [9]. The very idea of environment includes this notion of integration. This is clear in virtual campuses (see definition on page 3). Because of their broad scope, they have to fulfill administrative functions: managing who is registered to which courses, collecting assessment notes to count credits... Virtual places have to reproduce most functions which can be found on a real campus: registration, assistance, leisure \& fun... The integration of technologies is also present in smaller learning environments, for instance when a teacher sets up a page for a course that includes key pointers, a chat, a QUIZZ, a space to share drafts...

The word integrated refers to fairly different realities. There is a technical and a pedagogical integration, and both of them may vary in degree. The WEB technology has increased technical integration. At the lowest degree of integration, different pieces of software can be placed on the same Web page.

For instance, there is a small Author ware program running in the upper part of the page and a synchronous communication frame in the bottom of the screen in which the student can ask questions. The integration is here restricted to the fact that the two applications appear in the same window (versus in two different windows). Nevertheless, it is already interesting to design such a structured interface and not simply to ask the user to start multiple applications and configure the Screen.

A higher degree of integration is reached when applications share or exchange data structures. Examples:
If the student press 'help' in the Author ware program or if the program itself finds out that the student needs help, it opens up the chat and automatically sends to the teacher a request for help and a summary of what the student has done so far in the environment.

If the student enters an answer that the Author ware standard pattern matching techniques cannot parse, the message is passed to the mailer, which asks the teacher to provide feedback.

These examples show that the technical integration supports the pedagogical integration.

For instance, the designer has not to choose between self-instruction and tutoring, but decides to use both, self-instruction as the basis and tutoring when it is necessary [10]. For instance, micro worlds have often been criticized for a lack of coaching and information.

We can now have a chat within the micro world plus a rich hypertext (local and/or with pointers to Internet). For many years, the field of educational technology wad divided into schools of thoughts, e.g. Logo versus CBT. Now, that the designers can select the best of each approach, that using one technology does not exclude another one, the debate between these schools of thought will hopefully be recentered to understanding which types of interactions are relevant for which learning objectives.

\subsection{The virtual environment overlaps with the physical environment}

Virtual learning environments do not only integrate a variety of software tools but also integrate all the physical tools that can be found in a classroom. Of course, there exist some 'pure' virtual environments; designed for curricula that are completely at distance (Students never go to the school, never meet ...). But, most virtual learning environments include:

A variety of non-computerized learning resources: concrete manipulation tools, instruments, books, ... .

A variety of interactions that are not computermediated: face-to-face discussion among students, lectures by the teacher, group discussions..., plus traditional media such as letters, TV, phone and fax.

A variety of activities that are not computerbased: field trips, role playing, ... .

We will not enter here into the debate on what is virtual and what is not, on where the physical environment stops and where the virtual one starts. It is an interesting philosophical issue, more complex that the simple difference between computerized and Non-computerized elements. In the practice however there is no need to draw a boundary between physical and virtual worlds, the key is to integrate them, not to separate them. The continuity between physical and virtual objects becomes clear now that 
hybrid tools appear that connect computers with physical artifacts:

Boards on which students move blocks whose positions are known by the computers (as in chess games). These are physical objects, but coupled with a representation in the virtual space that can be used for interactions which are not possible in the physical world: computing all combinations of the elements assembled on the table, recording all the steps in assembling pieces, detecting where the current state of the artifact has already been reached before...

Single-display systems: several students interact live in front of same screen but with different mice22. While their actions are performed in the virtual space, most of their interactions occur in the physical world: the students not only talk to each other, but touch and even push each other.

\section{Fundamental Principles and Regulations in Designing the User Interface}

Good software will be viewed with an appropriate user interface. For designing an appropriate user interface we should consider a series of tested patterns, regulations and methods. Here, we will present some fundamental principles which have been studied and classified by skilled and professional software designers. Benefiting form these principles is a selective matter for designers according to their requirements which depends on the specifications of the designing software.

Here, we will present three golden rules in designing software [11, 12, and 13].

\subsection{First rule: considering the users as the controllers}

This rule relates to the process of controlling users in the user interface. In fact, the user who uses the software will control it. He controls the operation of the software considering its own requirements from it.

Based On this rule, represented descriptions, the principles extracted from the first rule can be considered as follows:

Using Correct Modes:

In designing the user interface should act in a way that uses recognized special modes and shapes in special places and positions. For example, the user interface which is used for playing music or audios instead of writing the word PLAY uses a recognized shape such as $\square$. This will make readability much easier for the users and will decrease the period of time required for thinking on the process of the operation. Because, this shape has been used repetitively in software as a symbol for PLAY and it has been internalized in the mind of the user. Texts:

Showing Appropriate Messages and Descriptive

Some of the users are interested to only use mouse for moving and working with the user interface and some of the other users are interested in both working with the keyboard and mouse. For giving more control to the users it is sufficient to make the possibility of special use of mouse and keyboard in the designed user interface to make it possible for the user to select between these two types of accesses. Thus, this possibility will make using it much easier.

Preparing Return Operation, Instantaneous and Their Reflections:

Fast responding to the requests of software should be appropriately applied in designing its interface to make it possible for the users to consider a better and more appropriate way for performing the operation by comparing the operation and studying its related reflections and reactions, for example using DEL in Command Prompt for omitting a file which will be instantaneously omitted.

Clarifying and Clarity in the User Interface:

The main purpose of the user interface is to help the users in order to make them feel that they do correctly and they are directly manipulating the objects with which they are working and in fact, such a state will be called the clarity of the interface.

Allowing the Users to Specify Their Own User Interface:

This is one of the most significant specifications of the user interface which completely lets the user to freely specify the appearance (color, font, show type, classifying), specifying the user behaviorally (premise operation, macros and buttons) and specifying interactive techniques (clicking, short-cut keys and the type of clicking) which will be represented to the customer according to the interests of the customer. The designers who work on designing the interfaces which have considered these specifications will experience more satisfaction and calmness because they benefit from their own patterns and interests in specifying the software.

Figure 1 an example of the site which has these specifications is represented. This site is OXFORD. By inspecting and observing this site more carefully we will discover that by entering this site the user will feel that he has this ability to specify the site according to his interests and this is exactly the first rule in designing the user interface. 


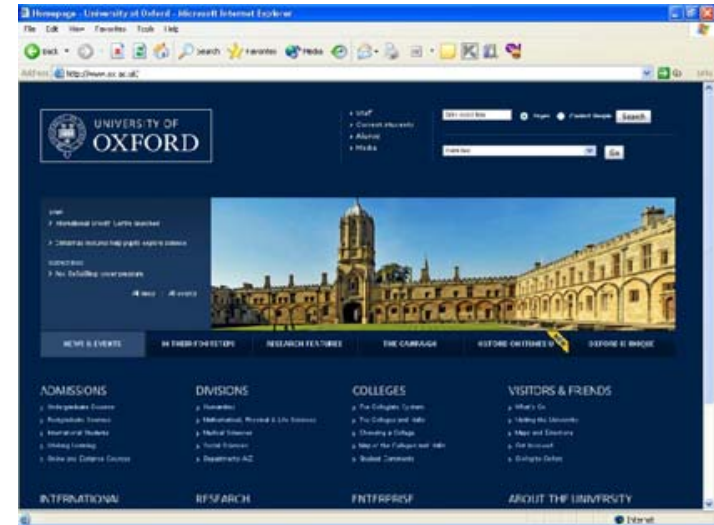

Figure 1. Oxford University Site

\subsection{Second rule: decreasing the cognition load of the users' memory}

The ability of the users in recognizing the operation of the user interface is different considering its symptoms in different people based on informing levels. In designing the user interface we attempt to make the user think contemplate less for recognizing the user interface and working with it. For this purpose some significant and effective principles have been studied in the following parts for decreasing the user's cognition load.

Making Words and Phrases Easier in the Mind:

If use some specified operations for a special operation their reminding in the mind will be done in a shorter period of time. This is one of the simple principles which most of the users of the user interfaces does not pay attention. Word and phrases such as copy and cut are internalized in the users' minds because they are applied in different software's and programs and they are significantly used in the body of the user interface.

Preparing Visual Points:

One of the important purposes of a graphical interface is that the users should know in what position they are placed, what they are doing and what they must do. Here for declining the duration of the process one of the prefabricated patterns are used which is determined by writing the first alphabets of the prefabricated information list in each field to make it easy for the user to choose his favorite issue in order to decline the duration of the user's cognition load.

Preparing the Premise, Redo and Undo:

By preparing open conditions for the users who specify the software according to their own interests, existence of some methods for converting the information to the past state or re-doing the specified operation is necessary.

Preparing the Short-Cut Key For the User Interface:

In addition to considering different states of applying mouse and keyboard in the software, using some methods for simplifying the number of clicking operations on keyboard and mouse and using the short-cut keys which decline the duration of the user's cognition period of time in the operation and the user will perform the mentioned operation by considering the text which has been written on the user interface in each operation and by using the help of Alt and Ctrl. For example HELP is performed by Alt $+\mathrm{H}$.

Increasing Clarification:

Users should not get deterred in what they can do in the product when facing the interface designing and there is no need to represent all the facilities and functions related to the user simultaneously. Users will have access to special capabilities of the user interface considering their requirements and the time they have access to them. Thus it is better to increase special facilities for the user by considering his profile and the type of his request. New interface technologies use wizards to help the users and increasing their facilities by representing them their required guidance.

According to the discussed issues, an example of the sites with these specifications is represented in Figure 2. This site is IBM. Simple designing is clearly obvious in this site. The user who faces this site for the first time doesn't spend a lot of time thinking on menus and links. So, in fact this site has observed the second rule represented in designing the user interface.

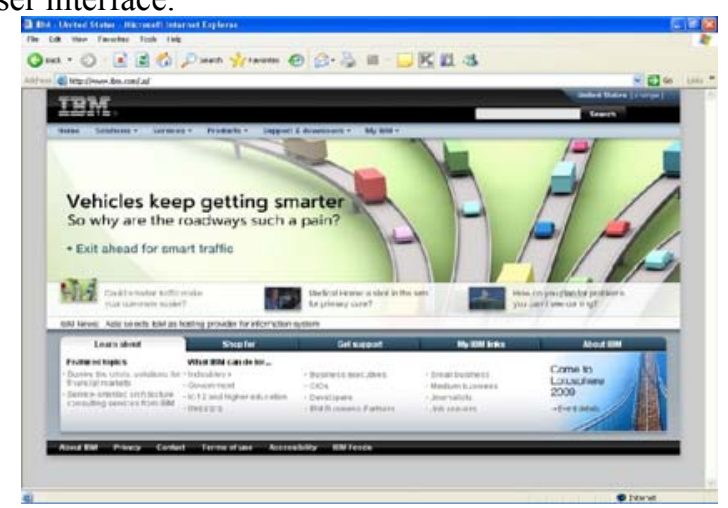

Figure 2. IBM Company

\subsection{The third rule: creating stability in the user interface}

Compatibility in designing the user interface is a fundamental key for applying the interfaces. This rule has less priority comparing with the two previous rules. Thus, in non sensitive environment it is not necessary to observe this rule. One of the best advantages of compatibility is that users can transfer their knowledge and educations of a new program in case that they are compatible with the current software. This rule includes a series of principles which are:

Applied Text Operation Stability: 
The user should be completely aware of him in software and for moving to different sections of the software. Thus, it is necessary to provide him with the ability to have a better and faster access to related sections by representing a general tree of the possible directions. Thus, considering this pattern in designing the user interface is highly useful and applicable.

Maintaining the Results of the Interactions and Similar Operations:

Using orders and functions which are designed in one section of the user interface must have similar results for other sections of the user interface, for example, if in one special form Esc key writes cancellation. This key must have the same meaning and application in other forms of the user interface.

Providing Beauty, Attractiveness and Unity in the Interface:

Using united colors, fonts and styles have special and significant effects on the appearance of the user interface. This means that the user will be satisfied by the software if the patterns are to be chosen according to the users' psychiatric interests.

\section{Suggestive user interface for e-learning Environment}

Education part of e-learning site which in fact is interactive approach of user and e-education is the most important part that registered users in system have a direct interaction to it. This part has several subsections, that due to subject of extend, only few main and necessary parts are discussed here:

Personal page: The first page user sees after entering and is very important in terms of design;

Virtual class: In fact is the main interactive tool between professor and student;

Communicative part: Allow participants to discuss and speak with each other simultaneously; and

Library: Available immediately books, articles, magazines and etc to users.

\subsection{Personal Page}

Student must sufficiently dominant to its demand and his/her personal page should present these facilities to them in best manner. In this section it should give full information about whole site to users, because of this, need a power user interface is highly feels. Presented suggestions in this part are first in whole site in particular this page Web2 and Ajax technology. Gadgets must be present by site manager in personal page in order to meet users' demand. Suggestive facilities of the above gadgets include:
1. Provide to use different sites' RSS, that user may use latest information and main scientific findings.

2. Unread messages show becomes in user e-mail.

3. Messages that have been messaged by other users through site locally become illustrative in this page.

4. Possible to show list of people in system at the same time (if mentioned person has activated this property), and while physical students and professors interaction in a virtual system is lower than verbal educational system, it is highly required to provide a live communication using modern programming and executive methods, as a result, message transfer with these online people by clicking their names must be observed;

5. Possible to choose pre-defined themes by site manager in order to avoid user's boring;

6. Educational calendar: The solar based calendar may use as main informative and reminding subjects of term. Thus, added event on calendar observes with different color and if mouse of computer clicks it, it shows descriptions of that event. It is noted that determined virtual classes date and time by professor also is presented in this calendar with different color. In addition, to close determined dates on calendar, its main events alarmingly on top of personal page after entering user may become illustrated and this event few hours before occurrence may send to user's fix line or mobile phone through short message or speaking (vocal) message;

7. Bulletin board: Required educational syllabuses of each course which should be informed to students of that course are placed in this bulletin board with professor's recognition (exactly like a bulletin board in verbal university). Also, this board must contain announced information (that considered user should be informed) by the university's instruction section. The information should be determined to user based on importance by professor or instruction section and be informed to user as well as educational calendar. As an example, if a class being cancelled with some reasons, the subject must be insert in bulletin board of students which have took this course credit, and if class cancellation is being occurred a day or few hours before participation, this subject must be informed to student through short message or vocal message;

8. Capability to show students' taken courses in current term;

9. May show other activities and facilities of site as a tree table to users (Site Map)

10. Questions (It will be explained in part 4.3)

Gadgets must be able to displace in whole personal page, size change and also deleting and adding by user. This helps considerably personal page simplicity and user memory reduction.

Such other facility that must be provided for virtual education site is a part called profile which 
user will fill it carefully, since helps largely in adequate user interface design. This profile should be performed intelligently, that, based on entered data and works user do during the time in addition to upgrade profile recognize users' interest and help to meet users' demand through performing their interest in user interface personal page. For instance, user interface to recognize what type of scientific data may user like and add similar information to gadget part of RSS section or implement user's interested themes in user personal page.

\subsection{Virtual Class}

E-learning is presented and performed in two independent complimentary spaces $[14,15]$ :

Unsynchronized learning space (Learning Management System).

Synchronized educational environment (talk room).

(1) Unsynchronized learning space is an environment in which you may (as a professor)

1. Present educational content to students.

2. Design tests for students and give them.

3. Observe and answer different related subjects to the course that address by students.

4. Follow up students' educational activities.

5. Determine exercises for students, evaluate their answers and mark them.

It may say this environment has been designed learning based.

(2) In synchronized educational environment, same as verbal class, a professor in a plan which considers earlier for the class:

1. Conduct students for learning a course subject.

2. Present subject.

3. Address questions and receive answers of students.

4. Students only see professor but professor sees all students.

5. Leave part of course or design of subjects and activities to students.

In fact, teaching in this space is professor based.

In synchronized education which is such virtual class, user interface must be designed not to feel virtually of this space so much by students and professors, and very close to real world. Although this realism may implemented in some ways: for instance, using 3D glasses in such way that imagine presence of all students in class or simulating class position in three dimensional, Dolby sound and 3D sound in the way that may feel professor's motion and displacement.

\subsection{Communicative Section}

Other point that has not been considered in educational environments is producing communicative protocol among professors and students in different universities in the world in which they become able to discuss and speak scientifically and consult with each other. This communicative protocol should be mentioned in user interface and be designed to provide text and voice discussion and file transfer with adequate speed for users. Helping from distributed architecture may lead to better production of this protocol.

Also adequate user interface design must be done for forum broadcasting. This part is about questioning between student and professor in scientific and course based subjects. Questions must be classified it means they are in which scientific field. These questions in addition to be taken in forum, they must with regard to students' interest that have been gathered by system during the time present in special gadget of questions for related student and students to answer these questions according to the interest.

\subsection{Library}

Digital library user interface must be designed in the way that may act beyond Rose net, that, in addition to indexed official books and articles in world's famous libraries and magazines, may provide a possibility to share scientific references which are in hand of students around the world. These references may contain thesis, course notes, researches and studies. Also copies of these information must be put in several servers to increase speed of access to the references.

\section{Evaluating suggestive user interface}

Suggestive user interface has been evaluated in software laboratory master part of department of computer in Qazvin Azad University. Students and professors referring to this lab voluntarily participated in user interface evaluation and completed polling based on provided fundamental principles and rules for user interface design. The

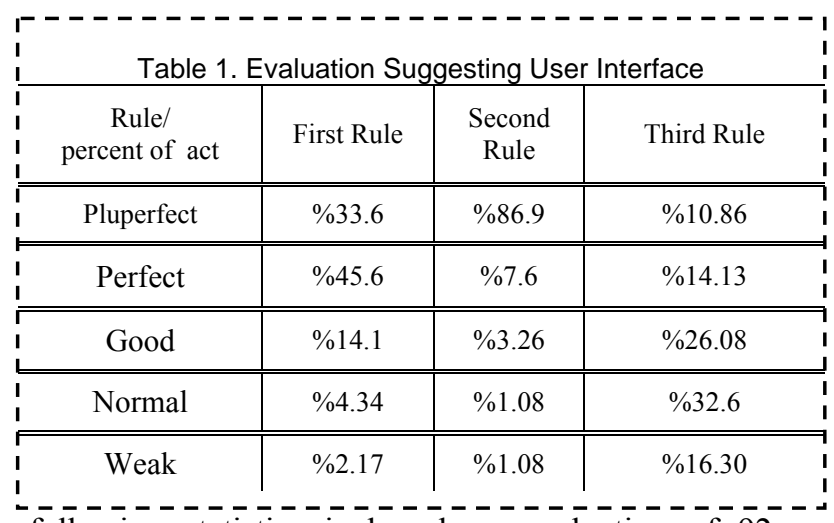

following statistics is based on evaluation of 92 students and professors which were participated in the polling. 
The statistics shows that second law implementation that is "reducing users' memory recognition pressure", has been applied significantly in suggestive user interface and also first law implementation which is "considering users as controllers", relatively is being approved by users, but unfortunately third law that is "stability in user interface" has not been almost successful in suggestive user interface. Although, as it said before, third law is less important than the two others.

\section{Conclusion}

User interface is the most important part of a software system or product and in fact is a window towards inside of software. In many cases, interface shows user's perception from system quality. If user interface is not designed based on presented principles and regulations, user may ignore to enter virtual education area which has many capabilities, and if doesn't ignore, a weak virtual education area with user interface may reduce strongly user's ability to apply power and capabilities of this system. In fact, weak interface leads a good design and its executive operation face fail position. Based on this, in this research an UI have been implemented and evaluated in Iran Azad University of Qazvin computer lab.

\section{References}

[1] Maguire, M. Elton, E. Osman, Z. Nicolle, C. (2006) 'Design of a Virtual Learning Environment for students with special needs', An Interdisciplinary Journal on Humans in ICT Environments, ISSN: 1795-6889, Volume 2 (1), 119-153.

[2] Reinecke K. Bernstein, A., ( 2008), 'Predicting User Interface Preferences of Culturally Ambiguous Users', ACM, 978-1-60558-012-8/08/04, Florence, Italy, April 5 April 10.

[3] Dillenbourg P., (2000), 'VIRTUAL LEARNING ENVIRONMENTS', Workshop on Virtual Learning Environments EUN conference.

[4] Dieberger A., (1999), 'Social connotations of space in the Design for Virtual Communities and Social Navigation', Social Navigation of Information Space, pp. 35- 54, Springer: London.

[5] Dourish P. Chalmers M., (1994), 'Running out of space: Models of Information Navigation', In Human Computer Interaction conference HCI, Glasgow.

[6] Munro A. Höök K. Benyon D., (1999), 'Footprints in the snow', Social Navigation of Information Space, pp. 114. Springer: London.
[7] Dourish P., (1999), 'Where the footprints Lead: Tracking down Other Roles for Social Navigation', Social Navigation of Information Space, pp. 15-34. Springer: London.

[8] Lehtinen E. Palonen, T., (1999) 'Evaluating CSCL Log Files by Social Network Analysi, Proceedings of the CSCL Conference, Stanford, pp.434-444, Dec.

[9] Peraya D. Piguet A. Joye F., (1999), 'Rapport d'information sur les mondes virtuels', Rapport rédigé pour loffice fédéral de la formation professionnelle et le la technique, Berne, Suisse.

[10] De Lièvre B., (1997), 'Une expérience d'encadrement en direct d'une formation pratique à distance', Actes du Colloque du Conseil Interinstitutionnel pour la technologie éducative, Montréal, Octobre.

[11] Lauesen S., (2004), 'User Interface Design, A Software Engineering Perspective', Addison-Wesley.

[12] Bouras C. Philopoulos A., (2000), 'Distributed Virtual Learning Environment: a Web-based Approach', Department of Computer Engineering and Informatics, University of Patras.

[13] Mandel, T. (1997) 'The Elements of User Interface Design', Chapter 5, Book, John Wiley \& Sons.

[14] Barfield, L. (2004) 'The User Interface: Concepts and Design", Bosko Books.

[15] Christos Bouras, Alexandros Philopoulos, (2000), 'Distributed Virtual Learning Environment: a Web-based Approach',Department of Computer Engineering and Informatics, University of Patras. 